\title{
Toxicidad retiniana de los fármacos utilizados en dermatología. Cloroquina y derivados
}

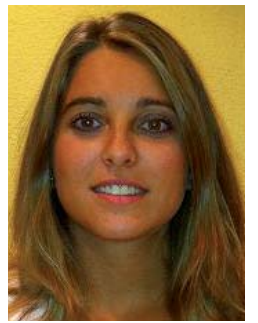

Lucía de Pablo Gómez de Liaño Médico residente de Oftalmología. Hospital Universitario 12 de Octubre. Madrid

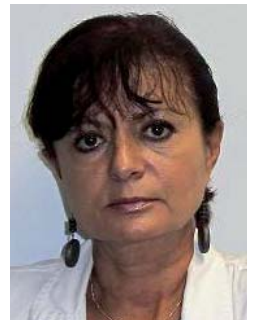

Isabel Redondo García Jefe de sección de Retina. Servicio de Oftalmología. Hospital Universitario 12 de Octubre. Madrid

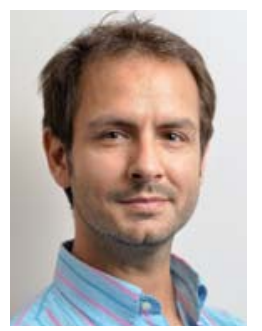

Jaime Campos Pavón Médico adjunto. Servicio de Oftalmología. Hospital Universitario 12 de Octubre. Madrid

\section{INTRODUCCIÓN}

Muchos de los fármacos utilizados para tratar enfermedades sistémicas pueden inducir efectos adversos oculares. Distintas clases de medicaciones pueden provocar reacciones adversas similares en el ojo y una sola medicación puede afectar a más de una estructura ocular. Es el caso de la cloroquina y sus derivados, tan utilizados en el campo de la dermatología.

Aunque la incidencia de toxicidad de estos fármacos es muy baja, resulta muy preocupante, porque la pérdida de agudeza visual asociada raras veces se recupera e incluso puede llegar a progresar después de la interrupción del fármaco.

Los pacientes y sus médicos deben ser plenamente conscientes de los riesgos oftálmicos y de la necesidad de exploraciones de cribado frecuentes para detectar la toxicidad retiniana en una fase temprana.

\section{CLOROQUINA Y DERIVADOS}

\section{Orígenes}

La cloroquina y la hidroxicloroquina son compuestos aromáticos del grupo 4-aminoquinolina, substitutos sintéticos de la quinina. El quino sigue siendo la única fuente útil de quinina; se trata de un árbol originario de
América, perteneciente al género Cinchona, de cuya corteza se extraen diversas sustancias, entre ellas la quinina.

La diferencia entre la cloroquina y la hidroxicloroquina radica en que la segunda se caracteriza por la b-hidroxilación de uno de los substitutos N-etil. Estas drogas fueron utilizadas inicialmente como antimaláricos durante la segunda guerra mundial. También se han usado para el tratamiento de la amebiasis extraintestinal y, por sus propiedades antiinflamatorias, han sido útiles en el manejo de algunos casos de artritis reumatoidea, lupus eritematoso discoide y lupus eritematoso sistémico (LES). Fue Payne quien usó por primera vez la quinina en 1894 para tratar el LES, al notar que dosis altas de este fármaco inducían palidez cutánea. En los años 1950, se popularizó el uso de los antimaláricos en enfermedades dermatológicas.

En 1959, Hobbs et al. reconocieron que el tratamiento prolongado con estas drogas inducía toxicidad retiniana ${ }^{1}$.

\section{Fisiopatología}

Tanto la cloroquina como la hidroxicloroquina tienen afinidad por la melanina, presente en la coroides, en el cuerpo ciliar y en el epitelio pigmentario de la retina (EPR) (capa 
más externa de la retina), donde se depositan. Antes de la alteración del EPR, se produce una alteración de la membrana citoplásmica de las células ganglionares de la retina y alteraciones degenerativas en los segmentos externos de los fotorreceptores (conos y bastones). Se ha demostrado también una disminución de la capa de fibras nerviosas del nervio óptico.

La cloroquina, al contrario que la hidroxicloroquina, altera la barrera hematorretiniana, por eso tal vez sea más tóxica. La incidencia de toxicidad retiniana es del $0,5-3,5 \%$ en pacientes tratados con hidroxicloroquina y del 10-25\% en el caso de la cloroquina ${ }^{2}$.

Histopatológicamente, existe una despigmentación del EPR, una pérdida de conos y bastones, y un acúmulo de pigmento subretiniano (fosfolípidos).

La retención prolongada de la droga en estos tejidos probablemente es la responsable de la progresión de la retinopatía o la aparición de la retinopatía de inicio tardío, a pesar de haberse suspendido la droga.

\section{Signos y síntomas oftalmológicos}

Depósito en forma de puntos blancos o líneas en espiral en el epitelio o estroma corneales (córnea verticilata; fig. 1), poliosis (alteración del color de las pestañas), disminución de la acomodación, parálisis de los músculos extraoculares, uveítis ante-

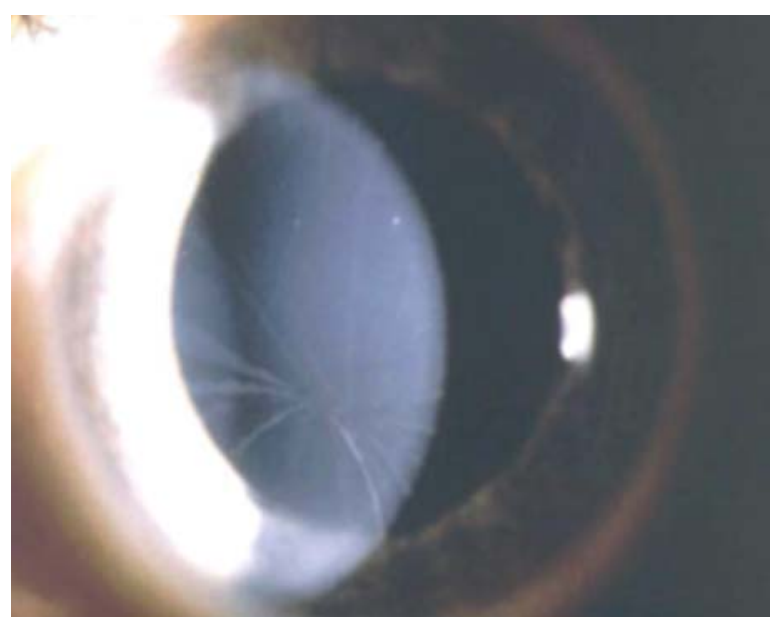

Figura 1. Córnea verticilata (líneas en estroma corneal). rior, catarata subcapsular, maculopatía en ojo de buey, neuritis óptica.

El paciente se queja de fotofobia, visión de flashes y disminución de la agudeza visual.

\section{Características de la maculopatía}

Los signos más tempranos incluyen cambios en el campo visual: escotoma paracentral, generalmente bilateral. Y alteración en el fondo de ojo: despigmentación granular sutil del EPR paracentral.

Con la exposición continua al fármaco, se produce la aparición progresiva de una maculopatía atrófica bilateral en diana (o en ojo de buey; fig. 2) que, en casos graves, se puede extender a todo el fondo de ojo, produciendo una atrofia retiniana generalizada y una gran pérdida de agudeza visual.

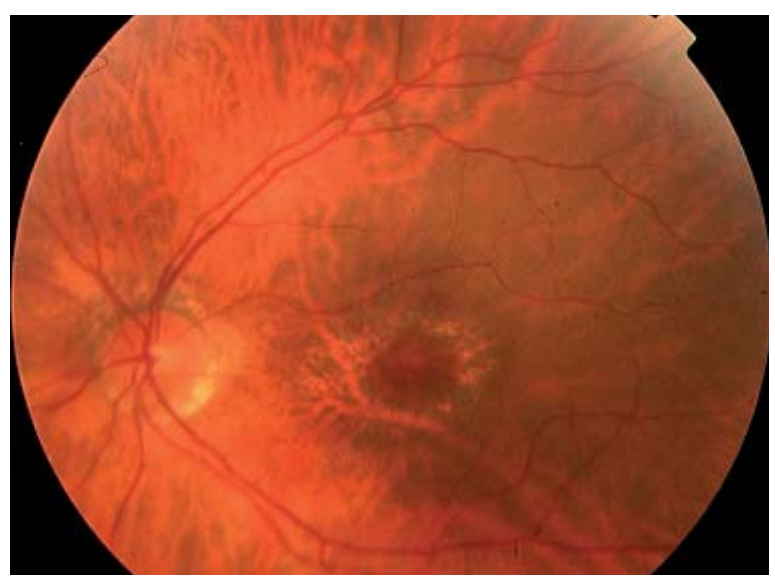

Figura 2. Imagen del fondo de ojo de la maculopatía en ojo de buey. Mujer de 57 años con lupus eritematoso sistémico en tratamiento con cloroquina $(250 \mathrm{mg} / \mathrm{d})$ durante 12 años.

Los estadios de evolución de la retinopatía aparecen recogidos en la tabla $1^{2}$.

\section{CRIBADO DE LA RETINOPATÍA}

\section{Criterios de riesgo ${ }^{3}$}

Se considera criterio de riesgo cuando se superan las siguientes dosis: $>3 \mathrm{mg} / \mathrm{kg} / \mathrm{d}$ de cloroquina o $>6,5 \mathrm{mg} / \mathrm{kg} / \mathrm{d}$ de hidroxicloroquina. 
Toxicidad retiniana de los fármacos utilizados en dermatología. Cloroquina y derivados

Tabla 1. Estadios de evolución de la retinopatía.

\begin{tabular}{|c|c|c|c|c|}
\hline Estadio & AV & CV & FO & \\
\hline Premaculopatía & Normal & $\begin{array}{c}\text { Alterado } \\
\text { (escotoma) }\end{array}$ & Normal & $\begin{array}{l}\text { El escotoma puede revertir } \\
\text { si se suspende el tratamiento }\end{array}$ \\
\hline $\begin{array}{l}\text { Maculopatía } \\
\text { establecida }\end{array}$ & $0,6-0,5$ & Alterado & $\begin{array}{c}\text { Halo parafoveal discreto } \\
\text { y palidez del EPR }\end{array}$ & $\begin{array}{c}\text { La interrupción del tratamiento } \\
\text { no revierte el escotoma, } \\
\text { pero evita progresión del cuadro }\end{array}$ \\
\hline $\begin{array}{l}\text { Maculopatía } \\
\text { en ojo de buey }\end{array}$ & $0,3-0,2$ & Alterado & $\begin{array}{l}\text { Hiperpigmentación foveolar } \\
\text { rodeada de zona de } \\
\text { hipopigmentación circundada } \\
\text { por anillo hiperpigmentado }\end{array}$ & $\begin{array}{l}\text { Puede evolucionar a pesar } \\
\text { del cese del tratamiento }\end{array}$ \\
\hline $\begin{array}{l}\text { Maculopatía } \\
\text { grave }\end{array}$ & $0,16-0,1$ & Alterado & $\begin{array}{l}\text { Pseudoagujero en fóvea, } \\
\text { extensa atrofia del EPR }\end{array}$ & $\begin{array}{l}\text { Puede evolucionar a pesar } \\
\text { del cese del tratamiento }\end{array}$ \\
\hline $\begin{array}{l}\text { Maculopatía en } \\
\text { estadio final }\end{array}$ & $<0,1$ & Alterado & $\begin{array}{c}\text { Gran atrofia del EPR, } \\
\text { estrechamiento arteriolar, } \\
\text { pigmentación retiniana periférica }\end{array}$ & $\begin{array}{l}\text { Puede evolucionar a pesar } \\
\text { del cese del tratamiento }\end{array}$ \\
\hline
\end{tabular}

AV: agudeza visual. CV: campo visual. FO: exploración de fondo de ojo.

Sin embargo, la literatura muestra que la dosis acumulada presenta datos predictivos más evidentes que la dosis diaria. La mayoría de pacientes que desarrollan toxicidad retiniana han recibido una dosis acumulada de cloroquina $>300$ $\operatorname{gramos}^{2,4}$.

También son criterios de riesgo: la duración del tratamiento $>5$ años, la presencia de enfermedad renal o hepática, la presencia de retinopatía concomitante y la edad mayor de 60 años.

\section{RECOMENDACIONES \\ DE LA AMERICAN ACADEMY OF OFTALMOLOGY (2002)}

\section{Examen inicial}

La agudeza visual, la exploración de la córnea con lámpara de hendidura, el fondo de ojo y el campo visual (mediante rejilla Amsler o Humphrey) son exploraciones que deben realizarse en todos los pacientes.

El test de colores, Ishihara o Farnsworth-Munsell, y la iconografía son opcionales, y la angiografía fluoresceínica (fig. 3) o el electrorretinograma multifocal se realizan de forma excepcional.

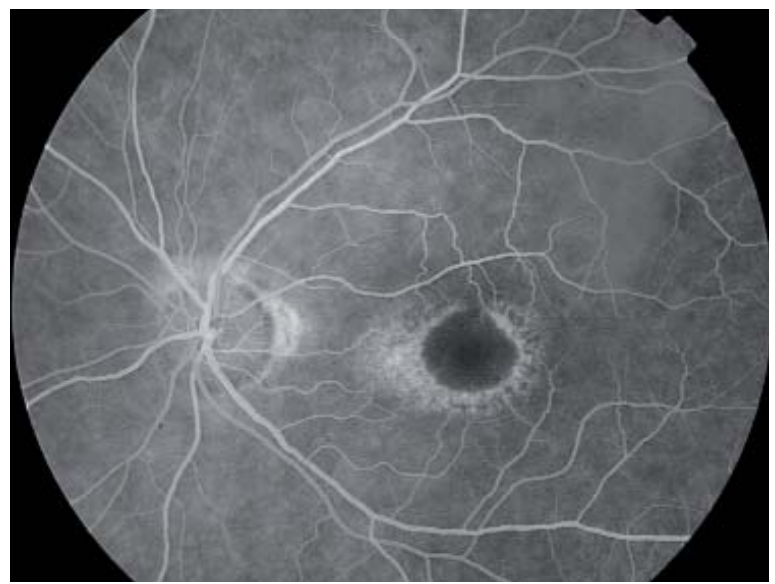

Figura 3. Angiografía fluoresceínica: anillo de hiperfluorescencia granular macular.

\section{En los pacientes de bajo riesgo}

Durante los primeros 5 años, se recomiendan las siguientes exploraciones, en función de la edad:

- 20-29 años: al menos un examen.

- 30-39 años: al menos dos exámenes.

- 40-64 años: cada 2-4 años.

- > 64 años: cada 1-2 años.

\section{En los pacientes de alto riesgo}

Se recomienda un examen anual. 
De Pablo Gómez de Liaño L et al. Toxicidad retiniana de los fármacos utilizados en dermatología. Cloroquina y derivados

\section{puntos clave}

- Se han demostrado efectos beneficiosos de los antimaláricos en pacientes de LES con manifestaciones cutáneas subagudas y crónicas, con respuesta adecuada entre el 50 y el $80 \%$ de los casos. Los proscritos con mayor frecuencia son la cloroquina $(250 \mathrm{mg} / \mathrm{d}$ ) y la hidroxicloroquina (200$400 \mathrm{mg} / \mathrm{d})^{5}$. Su uso, además, se extiende de forma compasiva a otro procesos, como la alopecia frontal fibrosante, el liquen, la fotosensibilidad, etc.

- La incidencia de toxicidad retiniana por antimaláricos es muy baja. Durante los estadios iniciales del uso del medicamento, la toxicidad es rara, la mayoría de pacientes que desarrolla retinopatía han usado la medicación durante más de 5 años.

- Se recomiendan exámenes oftalmológicos periódicos. El cribado se dirige a la detección temprana y la minimización de la toxicidad. Existe cierto grado de recuperación visual en estadios tempranos tras cesar el tratamiento.

- Se debe instruir a los pacientes en la utilización de la rejilla de Amsler para la automonitorización domiciliaria de forma frecuente.

- No existe otro tratamiento médico para la toxicidad de la cloroquina y la hidroxicloroquina que la finalización del tratamiento.

\section{En los pacientes con síntomas visuales sugestivos o hallazgos en el fondo de ojo}

Si el caso es dudoso, se debe revisar en 3 meses, mientras que si el diagnóstico está confirmado, se debe proceder a la retirada inmediata del antipalúdico, reevaluando a los 3 meses y al año siguiente.

\section{BIBLIOGRAFÍA}

1. Ferreras A., Pinilla I. Toxicidad retiniana secundaria a tratamiento con cloroquina. Arch Soc Esp Oftalmol. 2007;82:103-108.

2. Augusto $C$. Ocular complications of chloroquine and derivatives therapy. Arq Bras Oftalmol. 2010;73(4):384-9.

3. Warner A. Early hidroxicloroquine macular toxicity. Arthritis Rheum. 2001;44:1959-61.

4. Lai W, Lam B. Cloroquine-induced bull's eye maculopaty. Hong Kong Med J. 2005; 11:55-7.

5. Ramírez G, Gamarra G, Badillo R, Daza N, Uirbe BI. Lupus eritematoso sistémico. Guías de práctica clínica basadas en la evidencia [online]. p. 27. [Fecha de consulta: 25/10/2011]. Disponible en: http://www.bago.com/bolivia/html/doc_pdf/lupus.pdf 